\title{
Modeling of Hysteresis in Human Meridian System with Recurrent Neural Networks
}

\author{
Yonghong Tan, Ruili Dong and Hui Chen \\ College of Information, Mechanical and Electrical Engineering \\ Shanghai Normal University
}

China

\section{Introduction}

In the theory of the traditional Chinese medicine, it has been found that the acupuncturepoints are distributed in the meridian system of the human body. Moreover, meridian system is an independent system which exists in the body parallel with neural systems and blood circulation systems (Tsuei 1998, Trentini et. al. 2005). The experimental results have shown that the meridian system has significant effect on human health (Tsuei, 1998). Based on the recent research results, it was illustrated that the meridian system had an architecture with many channels allowing the electrical signals passed through easily (Zhang et. al. 1999, Yang 1997). That could be used to explain why the acupuncture-therapy would treat some diseases in human body by implementing some stimulating signals on the related acupuncture points. The acupuncture points distributed in the meridian system possesses some distinctive ways for transferring signals and processing information including electrical information (Yang 1997).

Until today, there have been some research results on human meridian system focusing on the analysis of impedance on single acupuncture-point (Yamamoto and Yamamoto 1987, Yang 1997, Zhang et. al. 1999). However, the meridian system is, in fact, a network with several channels. In each channel, there are several acupuncture-points located along a curve. The experimental results demonstrated that there were some relations among those points in each channel. Therefore, the analysis just depending on the impedance of one single acupuncture-point would not reflect the main characteristic of the signal transmission in human meridian system. One of the options is to use an excitation signal to stimulate an acupuncture-point in a channel of the meridian. Then the corresponding response of another acupuncture-point in the same channel is measured. Thus, the signal transmission performance of the measured channel in the meridian can be evaluated. Moreover, the experimental results have demonstrated that the human meridian system is a dynamic system (Zhang et. al. 1999, Yang 1997, Wang et. al. 2009). In this case, the identification of the model to describe the dynamic behavior of the meridian is an efficient way for performance evaluation. Wang et. al. (2009) developed an auto-regressive and moving average model to describe the human meridian system. It fits the response well when the exciting signal with slow frequency and the input amplitude is rather small. However, when the frequency of the exciting input is higher or the amplitude of the exciting signal is larger, 
it will illustrate some nonlinear behavior. Thus, a nonlinear dynamic model should be considered to describe this system.

This chapter is organized as follows: In the $2^{\text {nd }}$ section of this chapter, a brief description on the scheme of experiments to obtain the response of acupoints in a channel of the human meridian system by stimulating the acupoint in the same channel. Then, in the 3rd section, the analysis of the hysteretic characteristic happened in the response between two acupuncture-points in the same channel is illustrated. In section 4, an expanded input space is constructed to transform the multi-valued mapping of hysteresis to a one-to-one mapping. Then the recurrent neural network is employed to model both dynamics and nonlinearity of the meridian system. As neural network is a non-convex system, it is often stuck in local minima during the training. Therefore, we proposed a dynamic neural network based model with extreme learning machine (ELM) (Huang et. al. 2006 and 2007) to model the dynamic behavior of the human meridian system in section 5 . In the scheme, the values of the parameters of the hidden neurons and the weights connecting the hidden neurons with the inputs are specified by random values. The feedback factors connecting the outputs of the hidden nodes with the inputs of the hidden neurons are constrained within the region between zero and one to guarantee the stability of the neural network. However, the weights which interconnect the output of the network and the output of the hidden neurons are determined by least squares (LS) algorithm. In this case, the training of the neural network becomes an optimization for a convex system. Hence, the very good modeling results are derived. The corresponding experimental results are presented in section 6. In the experiment, both traditional ELM neural network and the proposed recurrent version are implemented to model the hysteresis in meridian system. The model validation results have shown that the proposed method has led to much better modeling performance.

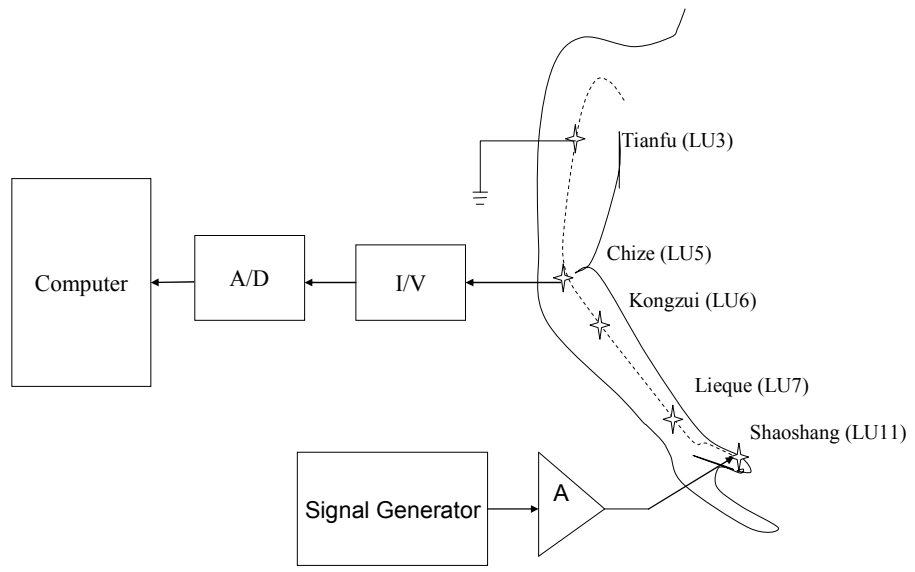

Fig. 1. Experimental Configuration of Measurement of Human Meridian Signal

\section{Brief description of the experimental configuration}

In this section, a method based on three detecting electrodes is used to measure both stimulation and the corresponding response of the acupuncture-points in meridian systems. 
The architecture of the measurement for meridian response is shown in Fig. 1. Based on the theory of Chinese medicine, there are 11 acupoints in the so-called Hand-Taiyin Lung Channel. In this experiment, the stimulating acupoint was Shaoshang (LU 11), the acupoint Tianfu ( $L U$ 3) was connected to ground, and the detecting acupoints were Chize (LU 5), Kongzui ( $L U$ 6) and Lieque ( $L U$ 7), respectively. The stimulation current signals were generated by a signal generator. Then, the signal was amplified by the amplifier A. In this experiment, Acupoint Shaoshang(LU11) was excited by the stimulation signals through a stimulating electrode. Then, the detecting electrode was used to measure the corresponding response of acupoint Chize(LU5) or the other acupoints in the same channel. The measured output was transferred through a current/ voltage conversion circuit then sampled by an analog / digital (A/D) convertor. The sampled signals were sent to the computer for further processing.

There were 6 healthy volunteers accepted the test. Before the test, the volunteers were relaxed to avoid the strenuous disturbance.

\section{The Response of the acupoint of the human meridian}

What the behavior of human meridian system to excitations would be? In this section, the experimental results will be presented to show the responses of the acupoint of the human meridian system to the excitation signals.

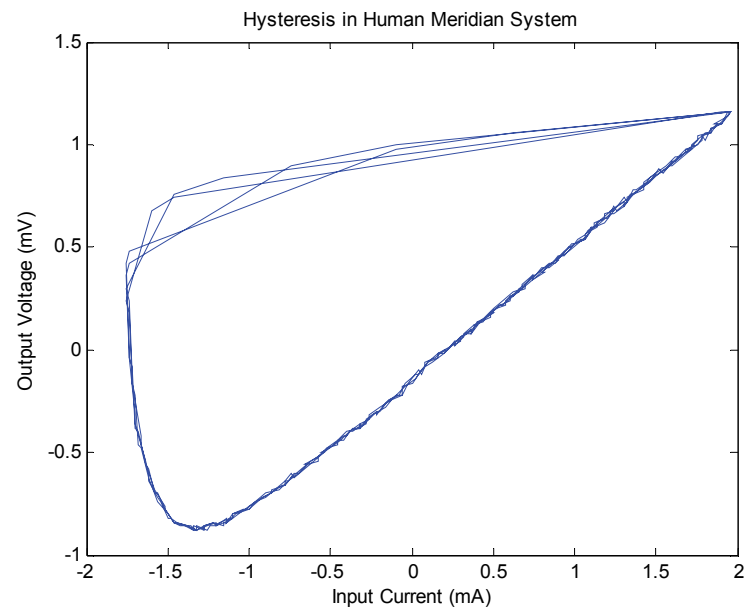

Fig. 2. The plot of the output of acupoint LU5 against the sawtooth input

In the experiments, we use a sawtooth waveform sequence to excite the meridian. A very interesting thing is that hysteresis phenomenon occurs in the human meridian systems. Fig. 2 shows the plot of the output of acupoint LU5 against the sawtooth waveform input. Obviously, the hysteresis presents asymmetric dynamic behavior in the response of the acupoint to the sawtooth waveform signal. During the upward segment, the output of the acupoint LU5 is almost a straight line. However, we can see the slower dynamics in the downward segment of the response. The asymmetric dynamic behavior implies the meridian is a nonlinear dynamic system. 
Moreover, From Fig. 2, we note that the hysteretic phenomenon happened in human meridian systems involved with the following characteristics:

1. non-smoothness;

2. multi-valued mapping;

3. asymmetry; and

4. slow time-varying.

Therefore, it is a dynamic system with rather complex characteristics. It is a real challenge to build a model to describe the meridian system with such comprehensive phenomena based on the conventional identification technique because the conventional identification methods can only be useful for the systems with smooth and one-to-one mapping. Hence, it is a challenge to construct a model of hysteresis.

\section{Modelling of hysteresis via Neural Networks}

Until today, there have been some modeling methods for hysteresis, e.g. Preisach model (Hu 2003) and Prandtl-Ishlinskii (PI) model (Macki et. al. 1993). Those methods used simple backlash operators as the basis functions for modeling. Therefore, lots of operators should be employed in order to obtain accurate models. Although there have been some modified Preisach model (Hu 2003, Ge and Jouaneh 1995 Ang et. al. 2003) and modified PI model (Dong and Tan 2010) proposed to describe the hysteresis systems, the structures of those modified schemes are still very complex. In order to simplify the architecture of the model to describe the behavior of hysteresis, Li and Tan (2004) as well as Zhao and Tan (2006) developed the so-called expanded input space based hysteretic models. In the expanded input space, a hysteretic operator which extracted the main movement feature of the hysteresis was introduced as one of the coordinates. Thus, the multi-valued mapping of hysteresis could be transformed to a one-to-one mapping between the inputs and output. Then, the feedforward neural networks were implemented to model the hysteresis based on the constructed expanded input space. However, due to the non-convex characteristics of the feedforward neural networks, one often met the problem that the training was easily stuck in local minima. It would have significant effect on the performance of the obtained neural models.

In this chapter, a modified scheme of the hysteretic operator given by Zhao and Tan (2006) is proposed. The modified hysteretic operator will handle the case of extreme-missing. Thus, a corresponding expanded input space is constructed. Then, the multi-valued mapping between the input and output of the hysteresis is transformed to a one-to-one mapping on this expanded input space. After that, the extreme learning machine (ELM) (Huang et. al. 2006 and 2007) is applied to the modeling of the hysteresis in human meridian systems.

In order to transform the multi-valued mapping of hysteresis to a one-to-one mapping, based on the expanded input space ( $\mathrm{Li}$ and Tan 2004, Zhao and Tan 2006), which is a twodimensional plane consisting of the input of hysteresis as well as the output of the hysteretic operator, is constructed. As the hysteretic operator can extract the movement tendency, such as increase, decrease and turning of the hysteresis, on this plane, the output of the hysteresis corresponding to the point in the input plane can be uniquely determined. One of the advantages of the expanded input space is that the one-to-one mapping between the input space and the output of the hysteresis can be constructed. Thus, the techniques for nonlinear modeling can be implemented to model the behavior of hysteresis on the constructed input 
space. Moreover, another advantage of this modeling method is that the computation of gradients at non-smooth points can be avoided.

Assumption: The considered hysteresis is continuous and forms a closed loop in the inputoutput diagram when the input cycles between two extrema.

Then, we define the hysteresis operator $f(x)$ as:

$$
f(x)=\left(1-e^{\left|x-x_{p}\right|}\right)\left(x-x_{p}\right)+f\left(x_{p}\right)
$$

where $x$ is the current input, $f(x)$ is the current output, $x_{p}$ is the dominant extremum adjacent to the current input $x . f\left(x_{p}\right)$ is the output of the operator when the input is $x_{p}$. The properties of the hysteretic operator are as follows:

1. Let $x(t) \in C\left(R^{+}\right)$, where $R^{+}=\{t \mid t \geq 0\}$ and $C\left(R^{+}\right)$is the sets of continuous functions on $R^{+}$. For the different time instance $t_{1}$ and $t_{2}$, it results in $t_{1} \neq t_{2}$ but $x\left(t_{1}\right)=x\left(t_{2}\right)$, where $x\left(t_{1}\right)$ and $x\left(t_{2}\right)$ are not the extrema. Then it leads to $f\left[x\left(t_{1}\right)\right] \neq f\left[x\left(t_{2}\right)\right]$.

2. If there exist two time instance $t_{1}$ and $t_{2}$, also $t_{1} \neq t_{2}$, such that $f\left[x\left(t_{1}\right)\right]-f\left[x\left(t_{2}\right)\right] \rightarrow 0$, then $x\left(t_{1}\right)-x\left(t_{2}\right) \rightarrow 0$.

Note that the hysteretic operator used by Zhao and Tan (2006) might encounter the problem that the output extreme of the hysteretic operator might be missed when the input just passes through the extreme. Therefore, in this section, a modified scheme of the hysteresis operator will be proposed.

Note that some extrema might be missed when the input just passed through its extrma. For example, suppose $t_{1}$ and $t_{2}\left(t_{1}<t_{2}\right)$ to be two time instances, in this case, if the corresponding values of the input at those two time instances are equal to each other, but the output of the hysteretic operator corresponding to one of the input value is in the increase zone while the output of the operator with respect to another input value is in the decrease zone. The extrema between those two output values of the hysteresis is obviously missed. To handle this problem, we have

Lemma 1: For the formula to describe the hysteresis operator shown in Eq. (1), if $x\left(t_{1}\right)=x\left(t_{2}\right), f\left[x\left(t_{1}\right)\right] \neq f\left[x\left(t_{2}\right)\right]$, where $t_{1}$ and $t_{2}$ are the adjacent time instances and $t_{1}<t_{2}$, the extrema located in the segment between points $\left(x\left(t_{1}\right), f\left(x\left(t_{1}\right)\right)\right)$ and $\left(x\left(t_{2}\right), f\left(x\left(t_{2}\right)\right)\right)$ can not be obtained within the time period $\left[t_{1}, t_{2}\right]$. However, it can be estimated by $\left(x_{m}, \operatorname{lm}\left(t_{2}, t_{1}\right)\right)$, where

$$
x_{m}=x\left(t_{2}\right)+0.5\left(x\left(t_{2}\right)-x\left(t_{1}\right)\right),
$$

and

$$
\operatorname{lm}\left(t_{2}, t_{1}\right)=\left\{\begin{array}{ll}
\left(1-e^{-x_{m}+x_{p}}\right)\left(x_{m}-x_{p}\right)+f\left(x_{p}\right), & x\left(t_{2}\right)>x\left(t_{1}\right) \\
\left(1-e^{x_{m}-x_{p}}\right)\left(x_{m}-x_{p}\right)+f\left(x_{p}\right), & x\left(t_{2}\right)<x\left(t_{1}\right)
\end{array} .\right.
$$

Proof: Suppose $x_{m}$ is the local maximum of the input, whilst $x\left(t_{1}\right)$ and $x\left(t_{2}\right)$ are located in the increase and decrease zones, respectively.

Hence, the derivatives of $f(x(t))$ with respect to $x(t)$ at $t_{2}$ and $t_{1}$ are

$$
\dot{f}\left(x\left(t_{2}\right)\right)=e^{-x\left(t_{2}\right)+x_{p}}
$$


and

$$
\dot{f}\left(x\left(t_{1}\right)\right)=e^{-x\left(t_{1}\right)+x_{p}}
$$

respectively.

Based on the assumption given by Zhao and Tan (2006), $\left(x_{p}, f\left(x_{p}\right)\right)$ is a local minimum, and $\left(x\left(t_{1}\right), f\left(x\left(t_{1}\right)\right)\right)$ is a point adjacent to the local maximum point $\left(x_{m}, \operatorname{lm}\left(t_{2}, t_{1}\right)\right)$. Hence, $\dot{f}\left(x\left(t_{2}\right)\right) \approx \dot{f}\left(x\left(t_{1}\right)\right)$ due to the properties of exponential function. That is to say, the three points, i.e. $\left(x\left(t_{1}\right), f\left(x\left(t_{1}\right)\right)\right),\left(x\left(t_{2}\right), f\left(x\left(t_{2}\right)\right)\right)$ and $\left(x_{m}, \operatorname{lm}\left(t_{2}, t_{1}\right)\right)$ can be considered to be approximately located in a straight line. Moreover, $\left(x_{m}, \operatorname{lm}\left(t_{2}, t_{1}\right)\right)$ is the point between $\left(x\left(t_{1}\right), f\left(x\left(t_{1}\right)\right)\right)$ and $\left(x\left(t_{2}\right), f\left(x\left(t_{2}\right)\right)\right)$, where $t_{1}$ and $t_{2}$ are the adjacent time instances. Define $L\left(t_{2}, t_{1}\right)$ represents the line connecting $\left(x\left(t_{1}\right), f\left(x\left(t_{1}\right)\right)\right)$ with $\left(x\left(t_{2}\right), f\left(x\left(t_{2}\right)\right)\right)$. So the extremum point in the space can be approximated to be the mean values of the projections of $L\left(t_{2}, t_{1}\right)$ on each coordinates in the plane. In other words, the mean value of the projection on the input coordinate is estimated by

$$
x_{m}=x\left(t_{2}\right)+0.5\left(x\left(t_{2}\right)-x\left(t_{1}\right)\right),
$$

and the mean value of the projection on the coordinate of the output of the hysteretic operator is estimated by

$$
\operatorname{lm}\left(t_{2}, t_{1}\right)=\left(1-e^{-x_{m}+x_{p}}\right)\left(x_{m}-x_{p}\right)+f\left(x_{p}\right) .
$$

Similarly, if $\left(x_{m}, \operatorname{lm}\left(t_{2}, t_{1}\right)\right)$ is a local minimum, $\operatorname{lm}\left(t_{2}, t_{1}\right)$ can be described by

$$
\operatorname{lm}\left(t_{2}, t_{1}\right)=\left(1-e^{x_{m}-x_{p}}\right)\left(x_{m}-x_{p}\right)+f\left(x_{p}\right) .
$$

Hence, combining Eqs.(4), (5) and (6) leads to

$$
\operatorname{lm}\left(t_{2}, t_{1}\right)=\left\{\begin{array}{ll}
\left(1-e^{-x_{m}+x_{p}}\right)\left(x_{m}-x_{p}\right)+f\left(x_{p}\right), & x\left(t_{2}\right)>x\left(t_{1}\right) \\
\left(1-e^{x_{m}-x_{p}}\right)\left(x_{m}-x_{p}\right)+f\left(x_{p}\right), & x\left(t_{2}\right)<x\left(t_{1}\right)
\end{array} .\right.
$$

Therefore, based on the above mentioned Lemma and the following Lemmas given by Zhao and Tan (2006), i.e.

Lemma 2: Let $x(t) \in C\left(R^{+}\right)$, where $R^{+}=\{t \mid t \geq 0\}$ and $C\left(R^{+}\right)$are the sets of continuous functions on $R^{+}$. If there exist two time instants $t_{1}, t_{2}$ and $t_{1} \neq t_{2}$, such that $x\left(t_{1}\right)=x\left(t_{2}\right)$, $x\left(t_{1}\right)$ and $x\left(t_{2}\right)$ are not the extrema, then $f\left[x\left(t_{1}\right)\right] \neq f\left[x\left(t_{2}\right)\right]$.

The details of the proofs can be referred to Appendix A.

Lemma 3: If there exist two time instants $t_{1}, t_{2}$ and $t_{1} \neq t_{2}$, such that $f\left[x\left(t_{1}\right)\right]-f\left[x\left(t_{2}\right)\right] \rightarrow 0$, then $x\left(t_{1}\right)-x\left(t_{2}\right) \rightarrow 0$.

The corresponding proof of lemma 3 can be referred to Appendix B.

Then, we have the following theorem, i.e.

Theorem: For any hysteresis satisfying Assumption, there exists a continuous one-to-one mapping $\Gamma: R_{2} \rightarrow R$, such that $F[x(t)]=\Gamma[x(t), f(x(t))]$. 
The detail of the proof can be referred to Appendix C.

It can also been proved that the obtained expanded input space is a compact set ( Zhao and Tan 2006) Hence, the mapping between the output and the input of the hysteresis on this expanded input space is a one-to-one mapping. Thus, the neural networks such as multilayer feedforward neural network can be implemented to model the performance of hysteresis on this input space.

\section{Recurrent ELM NN based model of hysteresis in human meridian systems}

Note the non-convex characteristic is one of the main drawbacks of the feedforward neural networks. It is often stuck in some local minima during the training procedure. Moreover, the slow convergence in training is kept it from the application in real-time cases. Huang et. al. (2006 and 2007) have proposed an efficient algorithm called as extreme learning machine (ELM) with the randomly specified input weights of the single hidden layered and the output weights of the network to be determined by using the least squares algorithm. ELM has achieved very good performance in generalization and much faster learning speed. The brief description of this neural network is presented as follows :

For $N$ samples $\left\{\left(x_{k}, t_{k}\right)\right\}_{k=1}^{N}$, where $\mathbf{x}_{k}=\left[x_{k 1}, x_{k 2}, \cdots, x_{k n}\right]$ is the $k$ th input vector and $\mathbf{t}_{k}=\left[t_{k 1}, t_{k 2}, \cdots, t_{k n}\right]$ is the $k t h$ target vector, a single layer feedforward network ( SLFN) with $\tilde{N}$ hidden neurons and activation function $g(x)$, i.e.

$$
\sum_{i=1}^{\tilde{N}} \boldsymbol{\beta}_{i} g\left(\mathbf{w}_{i} \cdot \mathbf{x}_{k}+b_{i}\right)=\mathbf{o}_{k}, k=1, \cdots, N ，
$$

where $\mathbf{w}_{i}=\left[w_{i 1}, w_{i 2}, \cdots, w_{i n}\right]^{T}$ is the weight vector connecting the $i$ th hidden neuron and the input neurons, $\boldsymbol{\beta}_{i}=\left[\beta_{i 1}, \beta_{i 2}, \cdots, \beta_{i m}\right]^{T}$ is the weight vector connecting the $i$ th hidden neuron and the output neurons, $\mathbf{o}_{k}=\left[o_{k 1}, o_{k 2}, \cdots, o_{k m}\right]^{T}$ is the output vector of the SLFN, and $b_{i}$ is the threshold of the $i$ th hidden neuron. Moreover, $\mathbf{w}_{i} \cdot \mathbf{x}_{k}$ denotes the inner product of $\mathbf{w}_{i}$ and $\mathbf{x}_{k}$. Hence, these $N$ equations can be written compactly as:

$$
\mathbf{H} \boldsymbol{\beta}=\mathbf{O},
$$

where $\mathbf{H}$ is called the hidden layer output matrix, i.e.

$$
\begin{gathered}
\mathbf{H}=\left[\begin{array}{ccc}
g\left(\mathbf{w}_{1} \cdot \mathbf{x}_{1}+b_{1}\right) & \cdots & g\left(\mathbf{w}_{\tilde{N}} \cdot \mathbf{x}_{1}+b_{\tilde{N}}\right) \\
\vdots & \cdots & \vdots \\
g\left(\mathbf{w}_{1} \cdot \mathbf{x}_{N}+b_{1}\right) & \cdots & g\left(\mathbf{w}_{\tilde{N}} \cdot \mathbf{x}_{N}+b_{\tilde{N}}\right)
\end{array}\right]_{N \times \tilde{N}}, \\
\boldsymbol{\beta}=\left[\begin{array}{c}
\boldsymbol{\beta}_{1}^{T} \\
\vdots \\
\boldsymbol{\beta}_{\tilde{N}}^{T}
\end{array}\right]_{\tilde{N} \times m}
\end{gathered}
$$

and 


$$
\mathbf{O}=\left[\begin{array}{c}
\mathbf{o}_{1}^{T} \\
\vdots \\
\mathbf{o}_{N}^{T}
\end{array}\right]_{N \times m} .
$$

By randomly assigning and fixing the input weights $\mathbf{w}_{i}$ and biases $b_{i}$, only leaving output weights $\boldsymbol{\beta}_{i}$ to be adjusted according to the objective function:

$$
\min _{\beta}\|\mathbf{H} \boldsymbol{\beta}-\mathbf{T}\|^{2}
$$

Based on theorem shown in section 4, the behavior of the hysteresis inherent in meridian system can be modeled by the ELM method on the obtained expanded input space. Therefore, the corresponding ELM based model for hysteresis is shown as follows:

$$
\begin{aligned}
\Gamma[x, f(x)] & =\mathbf{H}[x, f(x)] \boldsymbol{\beta}+\boldsymbol{\varepsilon} \\
= & {\left[\begin{array}{ccc}
g\left(\mathbf{w}_{1} \cdot\left[\mathbf{x}_{1}, f\left(\mathbf{x}_{1}\right)\right]+b_{1}\right) & \cdots & g\left(\mathbf{w}_{\tilde{N}} \cdot\left[\mathbf{x}_{1}, f\left(\mathbf{x}_{1}\right)\right]+b_{\tilde{N}}\right) \\
\vdots & \cdots & \vdots \\
g\left(\mathbf{w}_{1} \cdot\left[\mathbf{x}_{N}, f\left(\mathbf{x}_{N}\right)\right]+b_{1}\right) & \cdots & g\left(\mathbf{w}_{\tilde{N}} \cdot\left[\mathbf{x}_{N}, f\left(\mathbf{x}_{N}\right)\right]+b_{\tilde{N}}\right)
\end{array}\right]\left[\begin{array}{c}
\boldsymbol{\beta}_{1}^{T} \\
\vdots \\
\boldsymbol{\beta}_{\tilde{N}}^{T}
\end{array}\right]+\boldsymbol{\varepsilon}^{\prime} }
\end{aligned}
$$

where $\varepsilon$ is the modeling error vector, for any given $\varepsilon_{N}>0,\|\varepsilon\| \leq \varepsilon_{N}$.

By observing the response of the acupoints of human meridian systems, we can see that the dynamics in involved in the hysteresis. In order to describe the dynamic phenomenon of the hysteresis in meridian system, an internal feedback connection is introduced for each hidden neuron in the ELM neural network. Thus, we obtain a so-called recurrent ELM neural network on the constructed expanded input space for hysteresis inherent in human meridian. For a single input-single output network, the corresponding architecture is shown in Fig. 3. Obviously, we have

$$
\boldsymbol{\Gamma}[x(t), f(x(t))]=\mathbf{H}[\mathbf{x}(t), f(\mathbf{x}(t)), \mathbf{z}(t-1)] \boldsymbol{\beta}+\boldsymbol{\varepsilon}
$$

where

$$
\begin{array}{r}
\boldsymbol{\beta}=\left[\begin{array}{c}
\boldsymbol{\beta}_{1}^{T} \\
\vdots \\
\boldsymbol{\beta}_{\tilde{N}}^{T}
\end{array}\right] \\
\text { and } \\
\mathbf{H}[\mathbf{x}(t), f(\mathbf{x}(t)), \mathbf{z}(t-1)]= \\
{\left[\begin{array}{cccc}
g\left(\mathbf{w}_{1} \cdot\left[\mathbf{x}_{1}(t), f\left(\mathbf{x}_{1}(t)\right)\right]+\alpha_{1} z_{1}(t-1)\right) & \cdots & g\left(\mathbf{w}_{\tilde{N}} \cdot\left[\mathbf{x}_{1}(t), f\left(\mathbf{x}_{1}(t)\right)\right]+\alpha_{\tilde{N}} z_{\tilde{N}}(t-1)\right) \\
\vdots & \cdots & \\
g\left(\mathbf{w}_{1} \cdot\left[\mathbf{x}_{N}(t), f\left(\mathbf{x}_{N}(t)\right)\right]++\alpha_{1} z_{1}(t-1)\right) & \cdots & g\left(\mathbf{w}_{\tilde{N}} \cdot\left[\mathbf{x}_{N}(t), f\left(\mathbf{x}_{N}(t)\right)\right]+\alpha_{\tilde{N}} z_{\tilde{N}}(t-1)\right)
\end{array}\right]}
\end{array}
$$

where $\alpha_{i}$ is the feedback factor with the value randomly assigned within $(0,1)$ to guarantee the stability of the model and 


$$
z_{i}(t)=g\left(\mathbf{w}_{i} \cdot\left[\mathbf{x}_{j}(t), f\left(\mathbf{x}_{j}(t)\right)\right]+\alpha_{i} z_{i}(t-1)\right), i=1, \ldots, N ; j=1, \ldots, \tilde{N}
$$

For the assigned matrix $\mathbf{H}[$.$] , its \mathbf{Q R}$ decomposition is a matrix of the form

$$
\mathbf{H}=\mathbf{Q R}
$$

where $\mathbf{R}$ is an upper triangular matrix and $\mathbf{Q}$ is an orthogonal matrix, i.e., one satisfying

$$
\mathbf{Q}^{\mathrm{T}} \mathbf{Q}=\mathbf{I}
$$

where $\mathbf{I}$ is the identity matrix. This matrix decomposition can be used to simplify the computation to determine the solution $\boldsymbol{\beta}$, i.e.

$$
\hat{\boldsymbol{\beta}}=\left(\mathbf{R}^{\mathrm{T}} \mathbf{R}\right)^{-1} \mathbf{R}^{\mathrm{T}} \mathbf{Q}^{\mathrm{T}} \boldsymbol{\Gamma} .
$$

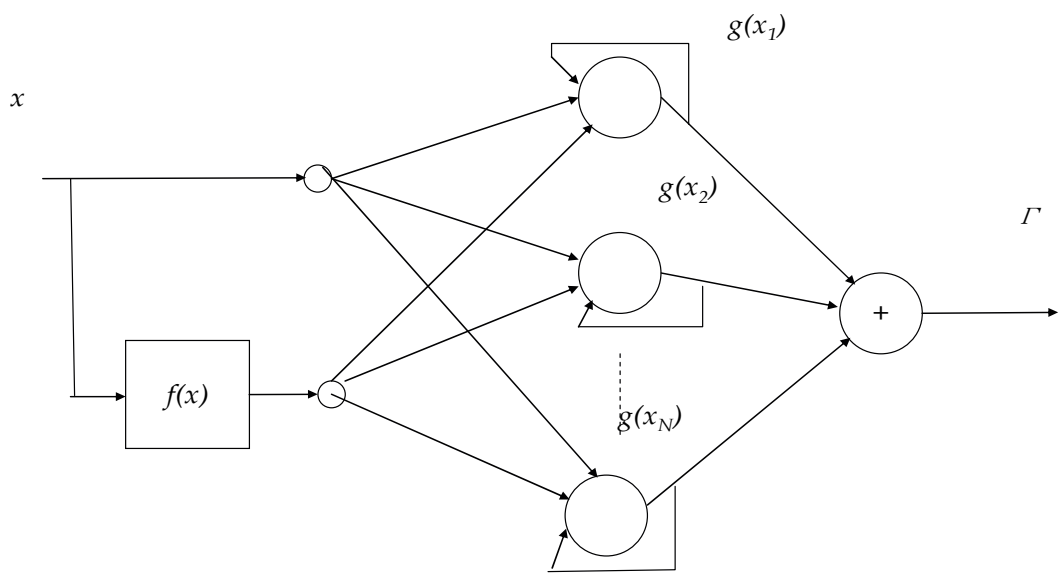

Fig. 3. The structure of recurrent ELM neural network

\section{Experimental results}

Based on the experimental setup shown in section 2, the experiment to measure the responses of acupoints was implemented. The semi-exponential signal was used to excite the meridian system. Both ELM neural network and recurrent ELM neural network were applied to modelling of the hysteresis happened in the meridian.

Firstly, we applied the ELM neural network with 30 hidden neurons to the modelling procedure of hysteresis. The $\mathrm{QR}$ decomposed least square algorithm was utilized to determine the weights of the neural model.

Fig. 4 shows the model validation result of the ELM neural network based model. It can be seen that the model failed to describe the hysteresis occurred in the meridian. On the other hand, we also applied the proposed recurrent ELM neural network on the constructed 
expanded input space. In the experiment, only 20 hidden neurons were involved in the network. The training algorithm to specify the weights of the model was the same as that in the training of ELM neural model. Fig. 5 illustrates the corresponding performance of model validation. Obviously, the proposed modelling method has achieved satisfactory result. Moreover, the proposed method has used less number of hidden neurons than that of the conventional ELM neural model. Thus, a much simpler model structure has been obtained.

\section{Conclusion}

A modeling method for hysteresis in human meridian systems is presented. In this modeling scheme, a modified hysteretic operator is proposed to construct an expanded input space to transform the multi-valued mapping of hysteresis into a one-to-one mapping. On the constructed expanded input space, the ELM neural network is employed to model the hysteresis inherent in human meridian systems. As the hysteresis in meridian system is an asymmetric and dynamic system, a recurrent ELM neural network is developed. In the proposed recurrent ELM neural network, the output state of each hidden neuron is fed back to its own input to describe the dynamic behavior of the hysteresis. The training of the recurrent ELM neural network is rather simple. A least square algorithm based on QR decomposition is implemented. The experimental results have shown that the proposed recurrent ELM neural model based model obtained much better modeling performance and simpler model structure.

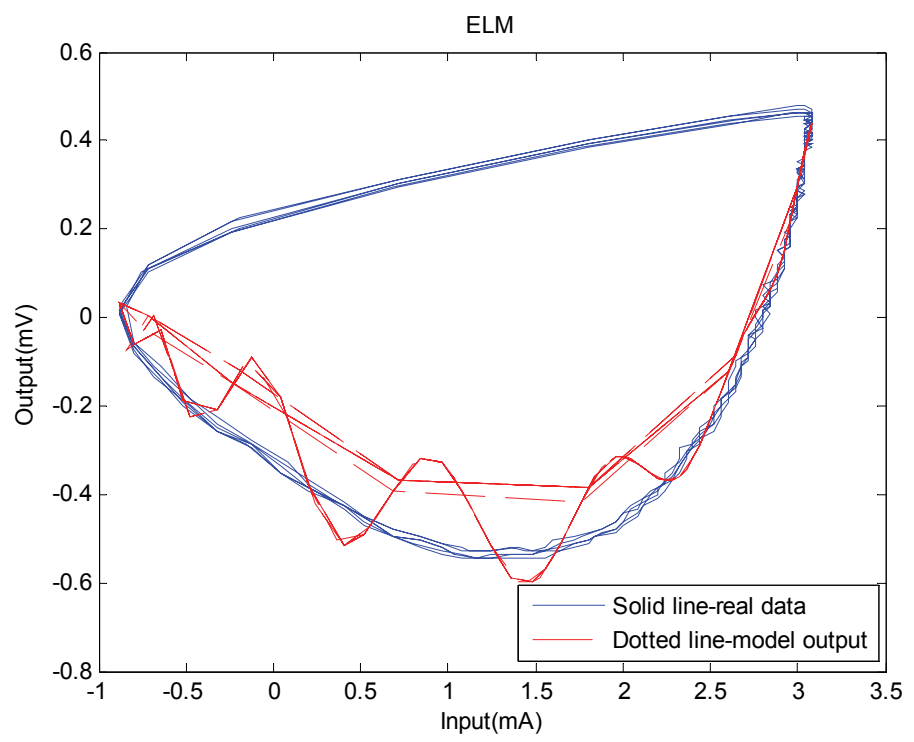

Fig. 4. Model validation of ELM neural network 


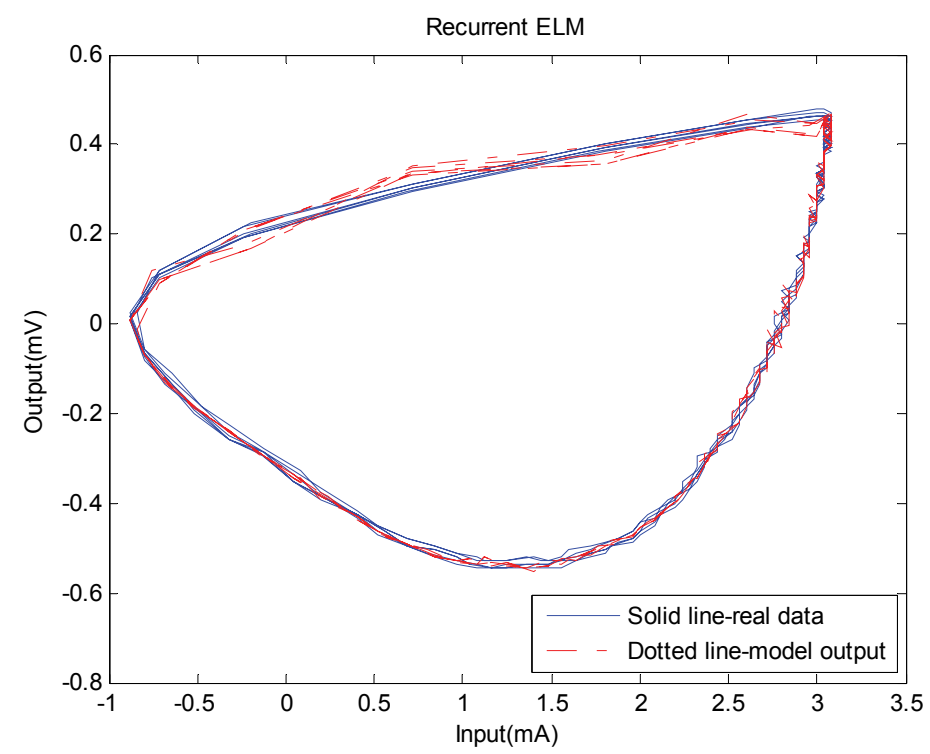

Fig. 5. Model validation of recurrent ELM neural network

\section{Appendix A}

The proof of lemma 2 is as follows (Zhao and Tan 2006):

Considering the segment $x(t)$ decreases monotonically, (1) becomes

$$
f_{d e}(x)=\left(1-e^{x-x_{p}}\right)\left(x-x_{p}\right)+f\left(x_{p}\right) \quad \dot{x}(t)<0
$$

where $f_{\mathrm{de}}(x)$ is decreasing segment of the function, $x_{\mathrm{p}}$ is the maximum extremum of the input. Whilst

$$
f_{\text {in }}(x)=\left[1-e^{-\left(x-x_{p}\right)}\right]\left(x-x_{p}\right)+f\left(x_{p}\right) \quad \dot{x}(t)>0
$$

denotes the increasing segment of the function. In this case, $x_{\mathrm{p}}$ is the minimum extremum of the input. Since

$$
\begin{gathered}
\frac{d f_{\text {in }}(x)}{d x}=e^{-\left(x-x_{p}\right)} \cdot\left(x-x_{p}\right)+\left[1-e^{-\left(x-x_{p}\right)}\right] \\
=1-\frac{1-\left(x-x_{p}\right)}{e^{x-x_{p}}}>1-\frac{1}{e^{x-x_{p}}}>0 .
\end{gathered}
$$

Therefore, $f_{\text {in }}(x)$ is monotonic. Similarly one can obtain that $f_{\text {de }}(x)$ is also monotonic. 


\section{Appendix B}

The following is the proof of lemma 3 (Zhao and Tan 2006).

Consider the case of $\dot{x}(t)>0$ that means in increase segment of the hysteresis. Assume that the segment $x(t)$ increases monotonically. From the proof of Lemma 1, it follows that

$$
f_{\text {in }}(x)=\left[1-e^{-\left(x-x_{p}\right)}\right]\left(x-x_{p}\right)+f\left(x_{p}\right)
$$

is monotonic as $x\left(t_{1}\right)-x\left(t_{2}\right) \rightarrow 0$. Thus, it yields

$$
f_{\text {in }}\left[x\left(t_{1}\right)\right]-f_{\text {in }}\left[x\left(t_{2}\right)\right] \rightarrow 0 .
$$

In terms of the property of continuity of the inverse function, it yields

$$
f_{\text {in }}\left[x\left(t_{1}\right)\right]-f_{\text {in }}\left[x\left(t_{2}\right)\right] \rightarrow 0,
$$

thus $x\left(t_{1}\right)-x\left(t_{2}\right) \rightarrow 0$. Similarily, when

$$
\begin{gathered}
f_{d e}\left[x\left(t_{1}\right)\right]-f_{d e}\left[x\left(t_{2}\right)\right] \rightarrow 0, \text { it results in } \\
x\left(t_{1}\right)-x\left(t_{2}\right) \rightarrow 0 .
\end{gathered}
$$

\section{Appendix C}

Firstly, it is proved that $\Gamma$ is a one-to-one mapping.

Case 1: Assume that $x(t)$ is not the extremum. In terms of lemma1, if there exist two different time instances $t_{1}$ and $t_{2}$, then

$$
\left(x\left(t_{1}\right), f\left[x\left(t_{1}\right)\right]\right) \neq\left(x\left(t_{2}\right), f\left[x\left(t_{2}\right)\right]\right) .
$$

Therefore, the coordinate $(x(t), f[x(t)])$ is uniquely corresponding to hysteresis $H[x(t)]$.

Case 2: Suppose that $x(t)$ is the extremum. In this case, for two different time instances $t 1$ and $t 2$, there will be

$$
\left(x\left(t_{1}\right), f\left[x\left(t_{1}\right)\right]\right)=\left(x\left(t_{2}\right), f\left[x\left(t_{2}\right)\right]\right) .
$$

According to the property of the Preisach-type hysteresis, $H\left[x\left(t_{1}\right)\right]=H\left[x\left(t_{2}\right)\right]$. Then the coordinate $(x(t), f[x(t)])$ will be uniquely corresponding to hysteresis $H[x(t)]$.

Combining the above-mentioned two situations, it is obtained that $\Gamma$ is a one-to-one mapping. Next, it will be verified that $\Gamma$ is a continuous mapping.

According to [11],

$$
x\left(t_{1}\right)-x\left(t_{2}\right) \rightarrow 0 \Rightarrow H\left[x\left(t_{1}\right)\right]-H\left[x\left(t_{2}\right)\right] \rightarrow 0 .
$$

Then, considering lemma 3,

$$
f\left[x\left(t_{1}\right)\right]-f\left[x\left(t_{2}\right)\right] \rightarrow 0 \Rightarrow x\left(t_{1}\right)-x\left(t_{2}\right) \rightarrow 0 \Rightarrow H\left[x\left(t_{1}\right)\right]-H\left[x\left(t_{2}\right)\right] \rightarrow 0 .
$$


Therefore, it can be concluded that there exists a continuous one-to-one mapping $\Gamma: R^{2} \rightarrow R$ such that $H[x(t)]=\Gamma(x(t), f[x(t)])$.

\section{Acknowledgment}

This research is partially supported by the Leading Academic Discipline Project of Shanghai Normal University (Grant No.: DZL811), the Innovation Program of Shanghai Municipal Education Commission (Grant Nos.:09ZZ141 and 11YZ92), the Advanced Research Grant of Shanghai Normal University (Grant Nos.: DYL201005 and DRL904), the National Science Foundation of China (NSFC Grant No.:60971004), the Natural Science Foundation of Shanghai (Grant No.: 09ZR1423400) and the Science and Technology Commission of Shanghai Municipality (Grant No.: 09220503000 and 10JC1412200).

\section{References}

R Becker, M. Reichmanis, A. Marino, J. Spadaro. (1976). Electrophysiological correlates of acupuncture points and meridians. Psychoenergetic Syst, Vol.1, 105-12.

R. Dong and Y. Tan. (2009). Modeling Hysteresis in Piezoceramic Actuators Using Modified Prandtl- Ishlinskii Model, Physica B, Vol.404, No.8-11, 1336-1342

P. Ge and M. Jouaneh. (1995), Modeling Hysteresis in Piezoceramic Actuators, Precision Engineering, Vol. 17, 211-221

G. Huang, Q. Zhu, C. Siew. (2006), Extreme Learning Machine: Theory and Application, Neurocomputing, Vol.70, 489-501

G. Huang, L. Chen. (2007). Convex Incremental Extreme Learning Machine, Neurocomputing, Vol.70, 3056-3062

H.Hu and R.Ben Mrad. (2003), On the Classical Preisach Model for Hysteresis in Piezoceramic Actuators, Mechatronics. Vol. 13, 85-94.

C. Li, Y. Tan. (2004). A Neural Networks Model for Hysteresis Nonlinearity, Sensors and Actuators A, Vol. 112, 49-54

J. W. Macki, P. Nistri, P. Zecca. (1993). Mathematical Models for Hysteresis , SIAM Review, Vol.35, No.1, 94-123

R. M, Marino AA, Becker RO. (1975). Electrical Correlatesof Acupuncture Points. IEEE Trans Biomed Eng, Vol.22, 533-5.

F. J. Trentini, B. Thompson and J. S. Erlichman. (2005). The Antinociceptive Effect of Acupressure in Rats, The American Journal of Chinese Medicine, Vol. 33, No.1,143-150

J. J. Tsuei. (1998). A Modern Interpretation of Acupuncture and the Meridian System. Proc. of the 2nd lntemational Conference on Bioelectromagnetism, 177 - 182

V. R. Twenty. (1975). Years of Electroacupuncture Diagnosis in Germany. A progress report. Am J Acupunct, Vol.;3, 7-17.

Z. Wang, Y. Tan, M. Su. (2009). Modeling of Meridian Channels, Proceedings of the International Conference on Biomedical Electronics and Devices, 167-172

Z. Wlodarski. (2005). Alternative Preisach Models, Physica B, Vol.367, 273-242

Y.Yamamoto and T. Yamamoto. (1987). Dynamic System for the Measurement of Electrical Skin Impedance. Med. Prog. Tech. Vol.12, 171-183

H.Y. Yang. (1997). The Research and Application of the Dynamic Testing System for Point Skin Resistance, Journal of Biomedical Engineering, Vol.16 No.1, 41-50 
W. Zhang, R, Xu and Z. Zhu. (1999). The Influence of Acupuncture on the Impedance measured by four electrodes on Meridians. Acupunct. \& Electro-Therap. Res. Int.J., Vol. 24, 181-188.

X. Zhao, Y. Tan. (2006). Neural Network Based Identification of Preisach-type Hysteresis in Piezoelectric Actuator Using Hysteretic Operator, Sensors and Actuators A, Vol. 126, 306-311 


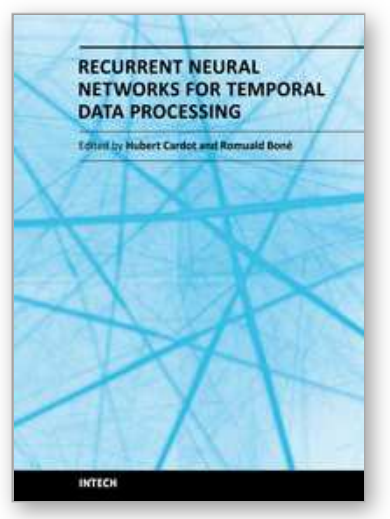

\author{
Recurrent Neural Networks for Temporal Data Processing \\ Edited by Prof. Hubert Cardot
}

ISBN 978-953-307-685-0

Hard cover, 102 pages

Publisher InTech

Published online 09, February, 2011

Published in print edition February, 2011

The RNNs (Recurrent Neural Networks) are a general case of artificial neural networks where the connections are not feed-forward ones only. In RNNs, connections between units form directed cycles, providing an implicit internal memory. Those RNNs are adapted to problems dealing with signals evolving through time. Their internal memory gives them the ability to naturally take time into account. Valuable approximation results have been obtained for dynamical systems.

\title{
How to reference
}

In order to correctly reference this scholarly work, feel free to copy and paste the following:

Yonghong Tan, Ruili Dong and Hui Chen (2011). Modeling of Hysteresis in Human Meridian System with Recurrent Neural Networks, Recurrent Neural Networks for Temporal Data Processing, Prof. Hubert Cardot (Ed.), ISBN: 978-953-307-685-0, InTech, Available from: http://www.intechopen.com/books/recurrent-neuralnetworks-for-temporal-data-processing/modeling-of-hysteresis-in-human-meridian-system-with-recurrentneural-networks

\section{INTECH}

open science | open minds

\section{InTech Europe}

University Campus STeP Ri

Slavka Krautzeka 83/A

51000 Rijeka, Croatia

Phone: +385 (51) 770447

Fax: +385 (51) 686166

www.intechopen.com

\section{InTech China}

Unit 405, Office Block, Hotel Equatorial Shanghai

No.65, Yan An Road (West), Shanghai, 200040, China

中国上海市延安西路65号上海国际贵都大饭店办公楼 405 单元

Phone: +86-21-62489820

Fax: $+86-21-62489821$ 
(C) 2011 The Author(s). Licensee IntechOpen. This chapter is distributed under the terms of the Creative Commons Attribution-NonCommercialShareAlike-3.0 License, which permits use, distribution and reproduction for non-commercial purposes, provided the original is properly cited and derivative works building on this content are distributed under the same license. 